\title{
Clinical features of catheter-related candidemia at disease onset
}

\author{
Yusuke $\underline{\text { Yoshino }}^{1}$, MD, Yoshitaka Wakabayashi ${ }^{1}$, MD, Satoshi Suzuki ${ }^{1}$, MD, Kazunori Seo ${ }^{1}$, MD, Ichiro Koga ${ }^{1}$, MD,
} Takatoshi Kitazawa ${ }^{1}$, MD, Shu Okugawa², MD, Yasuo Ota ${ }^{1}$, MD

INTRODUCTION Early detection of catheter-related candidemia is necessary to ensure that patients receive prompt and appropriate treatment. The aim of the present case-control study is to investigate the clinical features of catheter-related candidemia at disease onset, so as to determine the clinical indications for empiric antifungal therapy.

METHODS All 41 cases of catheter-related candidemia from September 2009 to August 2011 at a teaching hospital were included in the present study. To determine the characteristics that were risk factors for developing catheter-related candidemia, we compared all cases of catheter-related candidemia with all 107 cases of catheter-related blood stream infection (CRBSI) caused by non-Candida spp.

RESULTS In comparison with CRBSI due to non-Candida spp., the duration of catheter use was significantly longer in cases of catheter-related candidemia ( $13.9 \pm 9.0$ days vs. $23.2 \pm 25.2$ days). There was also a significant difference in the frequency of pre-antibiotic treatment between catheter-related candidemia and CRBSI due to non-Candida spp. (97.6\% [40/41 cases] vs. $44.9 \%$ [48/107 cases]). Patients with catheter-related candidemia also had significantly more severe clinical statuses (measured using the Sepsis-related Organ Failure Assessment score) than patients with CRBSI due to non-Candida spp. $(7.63 \pm 3.65$ vs. $5.92 \pm 2.81)$.

CONCLUSION When compared to patients with CRBSI caused by non-Candida spp., patients with catheter-related candidemia had significantly more severe clinical backgrounds, longer duration of catheter use and more frequent prior administration of antibiotic agents.

Keywords: candidemia, catheter-related bloodstream infection, clinical feature

\section{INTRODUCTION}

Infections of the bloodstream are serious and associated with high mortality, and catheter-related infections are a major cause of bloodstream infections. Catheter-related bloodstream infections (CRBSIs) are the main type of nosocomial infection and are largely attributable to Gram-positive microorganisms, such as Staphylococcus aureus and Staphylococcus epidermidis. These organisms have the ability to adhere to biomaterials and form a biofilm - an event that is generally believed to be key in the pathogenesis of CRBSI. Catheter removal and the prompt administration of appropriate antimicrobial agents are recognised as important therapeutic steps. ${ }^{(1)}$

Catheter-related candidemia is more serious than CRBSIs that are caused by other organisms; it is associated with higher mortality and requires longer treatment durations. ${ }^{(2)}$ Although immediate and appropriate antifungal treatment is important for improving the outcome of catheter-related candidemia, the initiation of antifungal agents tends to be delayed, resulting in the poor prognosis that is associated with the infection. ${ }^{(3)}$ Empiric antifungal therapy needs to be initiated immediately to improve outcome. Some studies have reported that empiric antifungal therapy for suspected catheter-related candidemia should be used in patients who have sepsis and any of the following risk factors: total parenteral nutrition, prolonged use of broadspectrum antibiotics, haematologic malignancy, receipt of bone marrow or solid organ transplant, femoral catheterisation, and colonisation by Candida spp. at multiple sites. ${ }^{(4,5)}$ However, those studies were carried out in limited situations, such as in a single cancer centre or a single intensive care unit. ${ }^{(4,5)}$ Further studies are needed to establish suitable indicators for initiating empiric antifungal therapy for CRBSI.

In the present study, we investigated the clinical features of catheter-related candidemia, with the aim of identifying novel and specific characteristics that would enable clinicians to distinguish between catheter-related candidemia at disease onset and CRBSIs due to non-Candida spp.

\section{METHODS}

The study population of the present study consisted of all patients who had catheter-related candidemia or bacteraemia due to non-Candida spp. during their stay in the University of Teikyo Hospital, Japan, a teaching hospital with 1,200 beds, from September 2009 to August 2011. The study was approved by the ethical committee of the University of Teikyo, Japan. In our institute (i.e. the University of Teikyo Hospital), administration of antifungals is not recommended in patients suspected to have CRBSI.

In the present study, the characteristics of catheter-related candidemia were compared with those of non-Candida CRBSIs. CRBSI was defined according to published guidelines. ${ }^{(1)}$ A patient was considered CRBSI-positive if at least one blood culture and catheter culture was positive for microorganisms, and the

${ }^{1}$ Department of Internal Medicine, Teikyo University School of Medicine, ${ }^{2}$ Department of Infection Control and Prevention, The University of Tokyo Hospital, Tokyo, Japan Correspondence: Dr Yusuke Yoshino, Assistant Professor, Department of Internal Medicine, Teikyo University School of Medicine, 2-11-1 Kaga, Itabashi-ku, Tokyo 173-8606, Japan. yoshinosuke0618@gmail.com 
patient displayed signs and symptoms of infection, including fever, chills, disorientation, hypotension, and respiratory failure, without other focal signs of infection. ${ }^{(1)}$ Elevated serum C-reactive protein (>0.3 mg/dL) and white blood cell count $\left(>12,000 / \mathrm{mm}^{3}\right)$ in the peripheral blood were useful adjuncts to the diagnosis of infection. The onset of CRBSI was defined as the day of the first positive blood culture sample. CRBSI cases that occurred $>30$ days after the initial episode were defined as new cases.

Blood specimens were inoculated into BACTEC ${ }^{\text {TM }}$ blood culture bottles (Becton, Dickinson and Company, Franklin Lakes, NJ, USA), and the blood cultures were assessed using the BACTEC ${ }^{\text {TM }}$ FX system (Becton, Dickinson and Company, Franklin Lakes, NJ, USA). If microbial growth was detected, yeast-like organisms were identified using the Analytical Profile Index identification system and ATB Expression ${ }^{\mathrm{TM}}$ system (bioMérieux, Lyon, France), while bacteria were identified using the WalkAway System (Siemens, Munich, Germany). The following antifungals were tested: fluconazole, voriconazole, micafungin and amphotericin B. Minimum inhibitory concentrations of antibacterial agents were determined via antimicrobial susceptibility assays using broth microdilution methods, according to the guidelines recommended by the Clinical Laboratory Standards Institute. ${ }^{(6,7)}$ Appropriate treatment was defined as the administration of suitable antimicrobial agents. ${ }^{(6,7)}$

The clinical courses of the patients were reviewed retrospectively to determine the following demographic characteristics: age, gender, severity of illness at disease onset, underlying factors (e.g. solid organ malignancy, haematologic malignancy, use of steroids or immunosuppressive agents, liver cirrhosis, history of surgery, and previous antibiotic use), bloodstream infection-attributable mortality, and previously demonstrated risk factors (e.g. total parenteral nutrition, prolonged use of broad-spectrum antibiotics, haematologic malignancy, receipt of bone marrow or solid organ transplant, femoral catheterisation, and colonisation due to Candida spp. at multiple sites). ${ }^{(4,5)}$

Patients who had undergone surgery at least once from birth prior to the onset of CRBSI were considered to have had a history of surgery. Previous antibiotic use referred to the use of antibiotics 14 days before the onset of CRBSI. Broad-spectrum antibiotics included third- and fourth-generation cephalosporins, carbapenems, quinolones, and beta-lactam/beta-lactamase inhibitor combinations. We defined prolonged use of broadspectrum antibiotics as $\geq 14$ days of administration of these antibiotic agents. The Sepsis-related Organ Failure Assessment (SOFA) score, ${ }^{(8)}$ which was used to measure the severity of infection, was calculated at the time of disease onset. CRBSIattributable mortality was defined as death that was considered to be caused by CRBSI within 30 days of disease onset.

The results of the present study are expressed as mean \pm standard deviation, unless otherwise indicated. Univariate analysis was performed using Student's $t$-test or Mann-Whitney $U$ test for continuous variables. Fisher's exact test was used, when appropriate, to compare proportions. Multivariate analysis was carried out via logistic regression analysis. All p-values were two-sided and $p$-value $<0.05$ was considered statistically significant. Statistical analyses were performed using Stat Flex version 6.0 (Artec Co Ltd, Osaka, Japan).

\section{RESULTS}

A total of 41 cases of catheter-related candidemia were recorded at our hospital during the study period. The Candida spp. identified included Candida albicans $(\mathrm{n}=21)$, Candida parapsilosis $(\mathrm{n}=12)$, Candida glabrata $(\mathrm{n}=6)$, Candida tropicalis $(\mathrm{n}=1)$, and Candida krusei $(\mathrm{n}=1)$. The 41 cases included in the present study involved 27 men and 14 women. The appropriate antifungal therapies were administered in 40 of the 41 cases $(97.6 \%)$, and the mean duration from disease onset to treatment initiation was $1.63 \pm 1.27$ days (for those 40 patients). Of the 41 patients, 17 (41.5\%) died of catheter-related candidemia despite the fact that most patients received appropriate antifungal treatments and the catheters were removed in all 17 patients.

The catheter-related candidemia cases were compared with non-Candida CRBSI cases in order to identify the significant clinical characteristics of catheter-related candidemia at disease onset (Table I). The results of our univariate analysis showed that patients with catheter-related candidemia had significantly more severe clinical backgrounds than patients with non-Candida CRBSI. The average SOFA score of patients with catheter-related candidemia was 1.71 points higher than that of patients with non-Candida CRBSIs. In addition, the duration of catheter use in patients with catheter-related candidemia was found to be significantly longer than that in patients with non-Candida CRBSIs. The median duration of catheter use in patients with candidemia was five days longer than that in patients with non-Candida CRBSIs. The frequency of the use of femoral catheters was also significantly higher in patients with catheter-related candidemia than in patients with non-Candida CRBSIs. There were also significant differences between catheter-related candidemia and non-Candida CRBSI in terms of the use of antibiotics before disease onset and prolonged treatment with broad-spectrum antibiotics. Of the six factors identified to be significant in the univariate analysis, only three (i.e. severe clinical background, antibiotic pretreatment and long duration of catheter use) remained significant features of catheter-related candidemia in the multivariate analysis.

\section{DISCUSSION}

The present study adds to the evidence of catheter-related candidemia being associated with high mortality. This association had been reported previously. ${ }^{(2)}$ Most cases of CRBSI that are caused by non-Candida spp., especially those caused by Staphylococcus spp., received appropriate empiric treatments at our institute. In contrast, not all cases of catheter-related candidemia received empiric antifungal therapies, and treatment was usually delayed. This delay in treatment might be a contributing factor to the high mortality of catheter-related candidemia.

In the present study, the following clinical characteristics were found to be significant risk factors for catheter-related candidemia: severe clinical background (i.e. high SOFA score), 
Table I. Characteristics of the patients with catheter-related infections (CRIs).

\begin{tabular}{|c|c|c|c|c|}
\hline \multirow[t]{2}{*}{ Characteristic } & \multicolumn{2}{|c|}{ No. (\%) } & \multirow{2}{*}{$\begin{array}{c}\text { Univariate } \\
\text { p-value }\end{array}$} & \multirow{2}{*}{$\begin{array}{c}\text { Multivariate } \\
\text { p-value }\end{array}$} \\
\hline & $\begin{array}{l}\text { CRI due to } \\
\text { Candida spp. } \\
(n=41)\end{array}$ & $\begin{array}{c}\text { CRI due to } \\
\text { non-Candida spp. } \\
\text { (n=107) }\end{array}$ & & \\
\hline Gender & & & 0.74 & \\
\hline Male & $27(65.9)$ & $71(66.4)$ & & \\
\hline Female & $14(34.1)$ & $36(33.6)$ & & \\
\hline Hospital stay* (days) & $48.5 \pm 43.5$ & $36.3 \pm 37.0$ & 0.09 & - \\
\hline SOFA score* & $7.63 \pm 3.65$ & $5.92 \pm 2.81$ & 0.003 & 0.02 \\
\hline Platelet concentration* $\left(\times 10^{4} / \mu \mathrm{L}\right)$ & $18.6 \pm 11.9$ & $18.7 \pm 12.0$ & 0.98 & - \\
\hline C-reactive protein concentration* $(\mathrm{mg} / \mathrm{dL})$ & $6.33 \pm 4.69$ & $6.93 \pm 6.16$ & 0.58 & - \\
\hline 30-day mortality & $17(41.5)$ & $16(15.0)$ & 0.001 & - \\
\hline \multicolumn{5}{|l|}{ Underlying factors } \\
\hline Solid organ malignancy & $12(29.3)$ & $31(29.0)$ & 0.97 & - \\
\hline Haematologic malignancy & $1(2.4)$ & $8(7.5)$ & 0.45 & - \\
\hline Liver cirrhosis & $6(14.6)$ & $6(5.6)$ & 0.09 & - \\
\hline Use of immunosupressive drugs & $11(26.8)$ & $20(18.7)$ & 0.28 & - \\
\hline History of surgery & $19(46.3)$ & $47(43.9)$ & 0.79 & - \\
\hline Receipt of bone marrow or solid-organ transplant & $0(0)$ & $2(1.9)$ & 0.60 & - \\
\hline \multicolumn{5}{|l|}{ Antibiotic treatment } \\
\hline Antibiotics pretreatment ${ }^{\ddagger}$ & $40(97.6)$ & $48(44.9)$ & $<0.0001$ & 0.0005 \\
\hline Prolonged use of broad-spectrum antibiotics & $12(29.3)$ & $7(6.5)$ & 0.0002 & 0.15 \\
\hline \multicolumn{5}{|l|}{ Catheterisation } \\
\hline Duration of catheter use $\mathrm{s}^{\S}$ (days) & $17(7-150)$ & $12(1-38)$ & 0.001 & 0.004 \\
\hline Femoral catheterisation & $8(19.5)$ & $8(7.5)$ & 0.03 & 0.61 \\
\hline Subclavian catheterisation & $1(2.4)$ & $8(7.5)$ & 0.25 & - \\
\hline Jugular catheterisation & $32(78.0)$ & $86(80.4)$ & 0.75 & - \\
\hline Total parenteral nutrition & $41(100.0)$ & $102(95.3)$ & 0.32 & - \\
\hline
\end{tabular}

Note: Non-Candida spp. consisted of the following microorganisms: Staphylococcus aureus $(\mathrm{n}=41)$, Staphylococcus epidermidis $(\mathrm{n}=35)$, Pseudomonas aeruginosa $(n=10)$, Enterococcus faecalis $(n=5)$, Acinetobacter baumannii $(n=3)$, Staphylococcus capitis $(n=3)$, Escherichia coli $(n=2)$, Bacillus cereus $(n=1)$, Enterobacter aerogenes $(n=1)$, Klebsiella oxytoca $(n=1)$, Klebsiella pneumoniae $(n=1)$, Morganella morganii $(n=1)$, Serratia marcescens $(n=1)$, Staphylococcus haemolyticus $(n=1)$, Staphylococcus lugdunesis $(n=1)$. *Data is presented as mean \pm standard deviation. ${ }^{\dagger}$ Sample size for CRI due to Candida spp. $=40$, while sample size for CRI due to non-Candida spp. $=105$. This is because one patient with CRI due to Candida spp. and two patients with CRI due to non-Candida spp. were undergoing catheter removal without antibiotic therapy at the discretion of the clinicians. All three patients recovered. ${ }^{\ddagger}$ Antibiotic pretreatment was defined as the use of antibiotics 14 days before the onset of CRBSI. §Data is presented as median (interval). SOFA: Sepsis-related Organ Failure Assessment

long-term use of a catheter, and antibiotic use before the onset of disease. A previous study, which was conducted in an intensive care unit setting, reported that the occurrence of fungal infection was associated with severe clinical backgrounds (e.g. severe injury, severe burns, and conditions that require mechanical ventilation). ${ }^{(9)}$ The results of the present study support the finding that a severe clinical background should raise suspicions of catheter-related candidemia, and therefore prompt the initiation of empiric antifungal agents.

A longer duration of catheter use is believed to be a clinical feature of catheter-related candidemia. This is because Candida infections are usually associated with the formation of biofilms on the surface of biological and inert surfaces, ${ }^{(10)}$ and the use of a catheter for a long period of time would provide a greater chance for the yeast to form a biofilm. Although some aspects of Candida biofilm formation remain unclear, it is possible that Candida spp. require a longer time to form biofilms as compared to bacteria. In the present study, the use of antibiotics before disease onset was also identified as a characteristic specific to catheter-related candidemia. This may be because antibiotic agents inhibit the proliferation of bacteria, but are not effective against Candida infections.

The following previously defined risk factors were only associated with catheter-related candidemia in our univariate analysis: prolonged use of broad-spectrum antibiotics, femoral catheterisation, and colonisation by Candida spp. at multiple sites. Other previously defined risk factors were not found to be associated with the occurrence of catheter-related candidemia in the present study (in both the univariate and multivariate analyses). This discrepancy may have been due to the retrospective and singlecentre nature of the present study and the present study's small study population. In addition, some of the previously documented risk factors were identified in small samples, such as patients in a single cancer centre or in the intensive care unit of a single hospital.

In conclusion, the results of the present study demonstrate that catheter-related candidemia is associated with a severe clinical background at disease onset, long-term use of a catheter, and antibiotic use before the onset of disease. Therefore, we recommend that empiric antifungal drugs be initiated in patients who display these clinical characteristics. 


\section{REFERENCES}

1. Mermel LA, Allon M, Bouza E, et al. Clinical practice guidelines for the diagnosis and management of intravascular catheter-related infection: 2009 Update by the Infectious Diseases Society of America. Clin Infect Dis 2009; 49:1-45.

2. Rex JH, Walsh TJ, Sobel JD, et al. Practice guidelines for the treatment of candidiasis. Infectious Diseases Society of America. Clin Infect Dis 2000; 30:662-78.

3. Morrell M, Fraser VJ, Kollef MH. Delaying the empiric treatment of candida bloodstream infection until positive blood culture results are obtained: a potential risk factor for hospital mortality. Antimicrob Agents Chemother 2005; 49:3640-5.

4. Lorente $L$, Jiménez A, Santana M, et al. Microorganisms responsible for intravascular catheter-related bloodstream infection according to the catheter site. Crit Care Med 2007; 35:2424-7.

5. Raad I, Hanna H, Boktour M, et al. Management of central venous catheters in patients with cancer and candidemia. Clin Infect Dis 2004; 38: 1119-27.
6. Clinical and Laboratory Standards Institute. Reference method for broth dilution antifungal susceptibility testing of yeasts; approved standard - third edition. Pennsylvania, USA: Clinical and Laboratory Standards Institute; 2008 Apr. Report no: M27-S4.

7. Clinical and Laboratory Standards Institute. Performance standards for antimicrobial susceptibility testing; twenty-first informational supplement. Pennsylvania, USA: Clinical and Laboratory Standards Institute; 2011 Jan. Report no: M100-S21.

8. Vincent JL, Moreno R, Takala J, et al. The SOFA (Sepsis-related Organ Failure Assessment) score to describe organ dysfunction/failure. On behalf of the Working Group on Sepsis-Related Problems of the European Society of Intensive Care Medicine. Intensive Care Med 1996; 22:707-10.

9. Tanaka H, Huruhata T, Gotou H, Sakurai M, Shimazaki S. [Fungal infection in patients with serious disease. Risk analysis of fungal infection]. Nihon Ishinkin Gakkai Zasshi 1999; 40:135-42.

10. Ramage G, Martínez JP, López-Ribot JL. Candida biofilms on implanted biomaterials: a clinically significant problem. FEMS Yeast Res 2006; 6:979-86. 